\title{
Plasma insulin and cardiovascular mortality in non-diabetic European men and women: a meta-analysis of data from eleven prospective studies
}

\author{
The DECODE Insulin Study Group
}

\begin{abstract}
Aims/hypothesis. We examined the association between plasma insulin and cardiovascular mortality in non-diabetic European men and women based on data from eleven prospective studies.

Methods. The study population comprised 6156 men and 5351 women aged 30-89 years. Baseline measurements included oral glucose tolerance test, fasting and 2-h plasma insulin, and conventional risk factors. Cox models were used to calculate hazard ratios (HRs) and their 95\% confidence intervals, and overall HRs were assessed by meta-analyses.

Results. During the 8.8-year follow-up, 362 men and 70 women died from cardiovascular disease. The ageand smoking-adjusted overall HR of cardiovascular mortality for the highest vs the lower quartiles of fasting insulin was 1.58 (95\% CI: 1.26-1.97) in men and 2.64 (1.54-4.51) in women. Adjusting for other risk factors in addition, the HR was $1.54(1.16-2.03)$ in
\end{abstract}

men and 2.66 (1.45-4.90) in women. For 2-h insulin these HRs were $1.28(0.99-1.66), 1.87$ (0.87-4.02), and $0.85(0.60-1.21), 1.36(0.53-3.45)$. The overall HRs for interquartile ranges for fasting and 2-h insulin, with full adjustment, were $1.13(1.05-1.22)$ and $1.11(1.01-1.23)$ in men, and $1.25(1.08-1.45)$ and $1.11(0.91-1.36)$ in women.

Conclusions/interpretation. Hyperinsulinaemia, defined by the highest quartile cut-off for fasting insulin, was significantly associated with cardiovascular mortality in both men and women independently of other risk factors. Associations between high 2 -h insulin and cardiovascular mortality were weaker and non-significant. Weak positive associations of fasting and 2-h insulin with cardiovascular mortality over interquartile ranges were, however, more similar.

Keywords Cardiovascular disease - Epidemiology · Hyperinsulinaemia $\cdot$ Insulin · Insulin resistance ·

Meta-analysis $\cdot$ Prospective studies $\cdot$ Risk factors $\cdot$ Sex
Received: 25 September 2003 / Accepted: 16 March 2004

Published online: 7 July 2004

C) Springer-Verlag 2004

Abbreviations: CVD, cardiovascular disease - DECODE, Diabetes Epidemiology: Collaborative analysis Of Diagnostic criteria in Europe · EGIR, European Group for the Study of Insulin Resistance - HOMA-IR, homeostasis model assessment of insulin resistance

Corresponding author of the DECODE Insulin Study Group: G. Hu, Diabetes and Genetic Epidemiology Unit, Department of Epidemiology and Health Promotion, National Public Health Institute, Mannerheimintie 166, 00300 Helsinki, Finland, Phone: +358-9-19127366, Fax: +358-9-19127313, e-mail: hu.gang@ktl.fi

Members of the DECODE Insulin Study Group are listed at the end of the paper

\section{Introduction}

The role of hyperinsulinaemia as an independent risk factor for cardiovascular disease (CVD) has previously been debated. An association between elevated plasma insulin, fasting or oral glucose load, and the risk of CHD or atherosclerotic CVD has been found in many $[1,2,3,4,5,6,7,8,9,10,11,12,13,14,15$, $16]$ but not in all $[17,18,19,20,21]$ prospective studies. Among the studies showing the positive association between plasma insulin and CVD, the effect of adjustment for other risk factors has varied. In several studies the association remained statistically significant, although attenuated $[1,2,3,5,6,7,9,10,12,13$, $15,16]$, whereas in other studies it became non-significant $[4,8,11,14]$. The majority of the published 
Table 1. Demographic characteristics of subjects, median duration of follow-up and number of cardiovascular deaths in eleven DECODE study cohorts

\begin{tabular}{|c|c|c|c|c|c|c|}
\hline \multirow[t]{2}{*}{ Study cohort } & \multirow[t]{2}{*}{$\begin{array}{l}\text { Number of } \\
\text { subjects }\end{array}$} & \multirow[t]{2}{*}{$\begin{array}{l}\text { Median age in } \\
\text { years (range) }\end{array}$} & \multirow[t]{2}{*}{ Men $(\%)$} & \multirow{2}{*}{$\begin{array}{l}\text { Median } \\
\text { duration of } \\
\text { follow-up } \\
\text { (years) }\end{array}$} & \multicolumn{2}{|c|}{$\begin{array}{l}\text { Number of cardio- } \\
\text { vascular deaths }\end{array}$} \\
\hline & & & & & Men & Women \\
\hline FINMONICA, Finland & 1626 & $54(44-64)$ & 45 & 8.9 & 25 & 5 \\
\hline Helsinki Policemen Study, Finland & 968 & $49(34-74)$ & 100 & 12.0 & 59 & \\
\hline East-West Study, Finland & 300 & $75(70-89)$ & 100 & 8.1 & 73 & \\
\hline Hoorn Study, The Netherlands & 2162 & $61(49-77)$ & 46 & 8.9 & 59 & 24 \\
\hline Zutphen Study, The Netherlands & 398 & 75 (69-89) & 100 & 4.7 & 42 & \\
\hline Newcastle Study, UK & 697 & $55(30-76)$ & 51 & 9.0 & 21 & 5 \\
\hline Goodinge Study, UK & 958 & $53(39-76)$ & 44 & 8.7 & 25 & 11 \\
\hline Ely Study, UK & 1006 & $54(40-69)$ & 43 & 11.8 & 14 & 5 \\
\hline Cremona Study, Italy & 1569 & $56(40-88)$ & 44 & 6.3 & 20 & 14 \\
\hline
\end{tabular}

studies were carried out in male populations, while some studies presented results for men and women combined. Therefore, data on the association between hyperinsulinaemia and CVD risk in women is scarce and conflicting. Six studies reported results for men and women separately $[2,8,10,12,17,19]$, but only two of them demonstrated the association between hyperinsulinaemia and CVD risk in women [8, 12].

Hyperinsulinaemia is the consequence of the underlying resistance of the tissues, mainly the skeletal muscle, to the action of insulin [22, 23]. Hyperinsulinaemia and insulin resistance are closely linked to several physiological and biochemical risk factors including overall and central obesity, impaired glucose regulation, dyslipidaemia characterised by high triglycerides and low HDL cholesterol, and elevated blood pressure [23]. A concept has been developed whereby this clustering of risk factors associated with Type 2 diabetes and CVD forms a distinct syndrome which has become known as "the insulin resistance syndrome", "the metabolic syndrome" or "the multiple metabolic syndrome" [24]. The upsurge in research and clinical interest in this field has refocused attention to the results of prospective studies on the association between plasma insulin levels and CVD risk, particularly as measurement of fasting insulin has been recommended as a surrogate estimate of insulin resistance in epidemiological studies [25]. In the present study we report results of meta-analyses of the association of fasting insulin and 2-h post-glucose insulin with cardiovascular mortality in non-diabetic European men and women based on data from eleven prospective studies. The main aims of our study were to examine whether the association between plasma insulin level and cardiovascular mortality is (i) similar for fasting insulin and 2-h insulin, and (ii) similar in men and women.

\section{Subjects and methods}

Study populations and baseline study methods. Centres in Europe that had performed population-based studies or large studies in occupational groups using the standard 2-h 75-g oral glucose tolerance test were invited to participate in the DECODE (Diabetes Epidemiology: Collaborative analysis Of Diagnostic criteria in Europe) study. The study populations and the methods used to recruit the participants have been described previously $[26,27,28,29,30]$. Individual data on fasting and 2-h glucose concentrations and a number of other variables were sent to the Diabetes and Genetic Epidemiology Unit of the National Public Health Institute in Helsinki, Finland for data analyses. The present study is based on data from eleven prospective studies, which are listed in Table 1. Eight of these studies had data for both men and women. All studies measured fasting plasma insulin at the baseline examination, and ten studies also measured 2-h insulin after an oral glucose tolerance test with $75 \mathrm{~g}$ glucose. All studies had baseline data on BMI, blood pressure, serum triglycerides or HDL cholesterol or both of these lipids, total cholesterol and smoking status, as well as follow-up data on CVD mortality. HDL cholesterol was not measured in the Helsinki Policemen Study. All investigations were carried out in accordance with the Declaration of Helsinki.

Because the DECODE studies started at different times independently of each other there was no standardisation of biochemical measurements between study centres. Thus, methods used for the measurement of plasma insulin were not uniform. The types of insulin assays used in the different studies are given in Table 2. Seven studies used conventional radioimmunoassays, which measure immunoreactive insulin and cross-react with proinsulin and its split products and are thus called "non-specific" for intact (true) insulin. Four studies used a radiometric assay or an ELISA that was "specific" for intact insulin. In our study we also used the score of homeostasis model assessment of insulin resistance (HOMA-IR; fasting insulin $[\mu \mathrm{U} / \mathrm{ml}] \times$ fasting plasma glucose $[\mathrm{mmol} / \mathrm{l}] / 22.5)$ as a surrogate measure for insulin resistance [31]. For statistical analyses, quartile cut-off points for fasting and 2-h insulin and HOMA-IR were calculated in each cohort for men and women separately. 


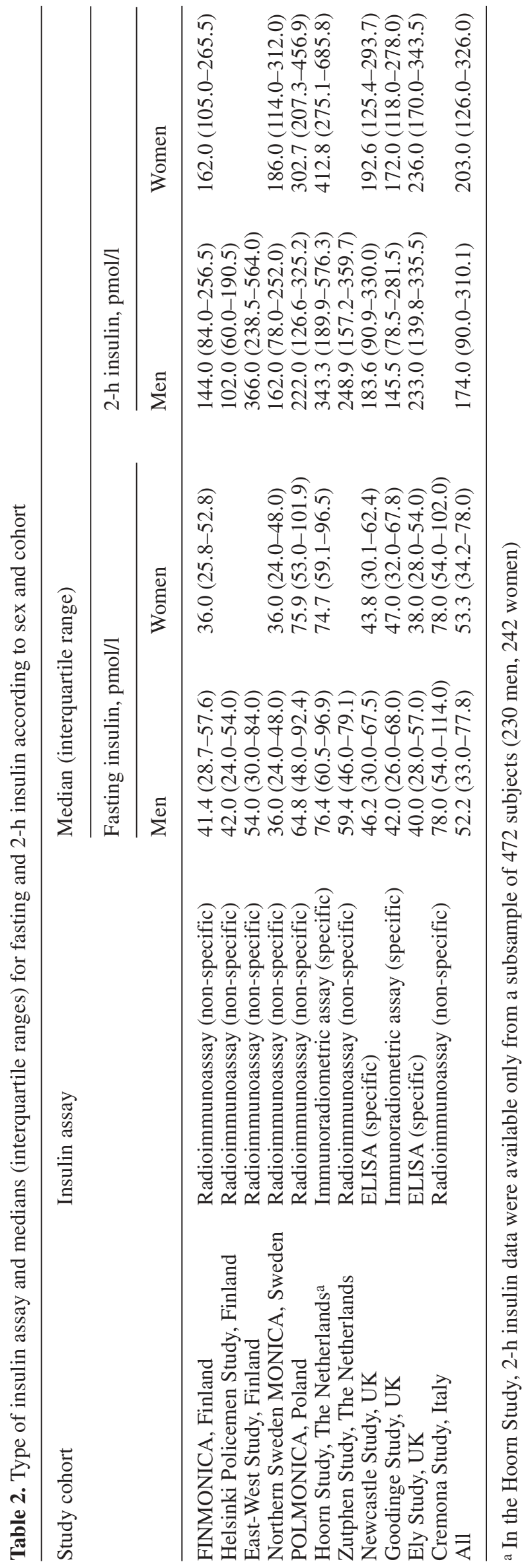

Subjects who had diabetes at baseline were excluded from the present analyses. For diagnosis, we used the 1999 WHO criteria [32]: previous diagnosis of diabetes or a fasting plasma glucose of $\geq 7.0 \mathrm{mmol} / \mathrm{l}$ and/or a 2-h plasma glucose of $\geq 11.1 \mathrm{mmol} / 1$.

Follow-up. Vital status was recorded for each subject who attended the baseline examination. Subjects who had emigrated and for whom vital status could not be confirmed were excluded from analyses at the time of emigration. The follow-up was almost complete (98-100\%) [28]. The Eighth, Ninth and Tenth Revisions of the International Classification of Diseases were used for coding the causes of death. The codes used for CVD were 401-448 (I10-I79).

Statistical analysis. Data analyses were performed using the SPSS for Windows 11.0 software. Associations of fasting and 2-h plasma insulin and HOMA-IR with other continuous risk factors were assessed by calculating Spearman's rank correlation coefficients, adjusted for age and cohort, in the pooled study population. The associations of conventional risk factor variables with cardiovascular mortality in men and women of the pooled study population were assessed by the Cox proportional hazards model. Age- and cohort-adjusted and multivariate-adjusted Cox model hazard ratios (HRs) and 95\% CIs with regard to the risk of cardiovascular mortality were calculated for each risk factor, separately for men and women. The relationship of fasting insulin, 2-h insulin and HOMA-IR with cardiovascular mortality was assessed by Cox models. Insulin variables and HOMA-IR score were entered into Cox models as dichotomous variables comparing the highest sex- and cohort-specific quartile for each variable with the respective combined quartiles 1-3. Cox model analyses were also performed by calculating HRs and 95\% CIs for sex- and cohortspecific interquartile ranges for fasting and $2-\mathrm{h}$ insulin. Cox models were adjusted for different sets of covariates including, as appropriate, age, smoking, BMI, systolic blood pressure, fasting glucose, 2-h glucose, total and HDL cholesterol, and triglycerides (log-transformed). In the pooled database analyses, the study cohort was also entered as a covariate. The possibility of a curvilinear association between insulin variables and cardiovascular mortality was tested by the log-likelihood ratio test comparing Cox models without and with squared term for the relevant insulin variable.

Meta-analyses were performed to assess the overall association of fasting insulin, 2-h insulin and HOMA-IR with cardiovascular mortality using a fixed-effects approach and the methods detailed by Fleiss [33]. The results are reported as overall HRs and 95\% CIs for cardiovascular mortality. In the meta-analyses of data on women, only the seven studies where at least four CVD deaths had occurred were included. A $p$ value of less than 0.05 was considered statistically significant.

\section{Results}

The study population comprised 6156 men and 5351 women aged 30-89 years. The number of subjects from each of the eleven study cohorts, and their demographic characteristics, are given in Table 1.

There were marked differences in the sex-specific medians and interquartile ranges of fasting insulin and 2-h insulin between cohorts (Table 2). The betweencohort variation in the ratio of the highest to the lowest quartile for fasting and 2-h insulin was, however, 
Table 3. Age- and cohort-adjusted Spearman's correlation coefficients of fasting insulin, HOMA-IR and 2-h insulin with other risk factors in the pooled data

\begin{tabular}{|c|c|c|c|c|c|c|c|c|c|}
\hline & BMI & $\begin{array}{l}\text { Systolic } \\
\text { BP }\end{array}$ & $\begin{array}{l}\text { Total } \\
\text { cholesterol }\end{array}$ & $\begin{array}{l}\text { HDL } \\
\text { cholesterol }\end{array}$ & $\begin{array}{l}\text { Triglyc- } \\
\text { erides }\end{array}$ & $\begin{array}{l}\text { Fasting } \\
\text { glucose }\end{array}$ & $\begin{array}{l}\text { 2-h } \\
\text { glucose }\end{array}$ & $\begin{array}{l}\text { Fasting } \\
\text { insulin }\end{array}$ & $\begin{array}{l}\text { HOMA- } \\
\text { IR }\end{array}$ \\
\hline \multicolumn{10}{|l|}{ Men } \\
\hline HOMA-IR & $0.34^{\mathrm{a}}$ & $0.09^{a}$ & $0.04^{\mathrm{a}}$ & $-0.20^{\mathrm{a}}$ & $0.22^{\mathrm{a}}$ & $0.21^{\mathrm{a}}$ & $0.14^{\mathrm{a}}$ & $0.98^{\mathrm{a}}$ & 1.00 \\
\hline 2-h insulin & $0.30^{\mathrm{a}}$ & $0.11^{\mathrm{a}}$ & $0.03^{a}$ & $-0.22^{\mathrm{a}}$ & $0.24^{\mathrm{a}}$ & $0.17^{\mathrm{a}}$ & $0.52^{\mathrm{a}}$ & $0.56^{\mathrm{a}}$ & $0.56^{\mathrm{a}}$ \\
\hline HOMA-IR & $0.37^{a}$ & $0.11^{\mathrm{a}}$ & $0.06^{\mathrm{a}}$ & $-0.23^{a}$ & $0.27^{\mathrm{a}}$ & $0.32^{\mathrm{a}}$ & $0.21^{\mathrm{a}}$ & $0.98^{a}$ & 1.00 \\
\hline 2-h insulin & $0.29^{a}$ & $0.17^{a}$ & $0.04^{\mathrm{a}}$ & $-0.23^{a}$ & $0.25^{\mathrm{a}}$ & $0.22^{a}$ & $0.46^{\mathrm{a}}$ & $0.54^{\mathrm{a}}$ & $0.57^{a}$ \\
\hline
\end{tabular}

a $p<0.05$; HOMA-IR, homeostasis model assessment of insulin resistance

Table 4. Univariate and multivariate Cox model hazard ratios (HRs) and their 95\% CIs for conventional risk factors with regard to cardiovascular mortality in the pooled data, shown according to sex (362 cardiovascular deaths among 6156 men and 68 cardiovascular deaths among 5183 women)

\begin{tabular}{|c|c|c|c|c|}
\hline \multirow[t]{2}{*}{ Risk factor } & \multicolumn{2}{|l|}{ Men } & \multicolumn{2}{|l|}{ Women ${ }^{\mathrm{a}}$} \\
\hline & $\begin{array}{l}\text { Univariate HR } \\
(95 \% \mathrm{CI})^{\mathrm{b}}\end{array}$ & $\begin{array}{l}\text { Multivariate HR } \\
(95 \% \mathrm{CI})^{\mathrm{c}}\end{array}$ & $\begin{array}{l}\text { Univariate HR } \\
(95 \% \mathrm{CI})^{\mathrm{b}}\end{array}$ & $\begin{array}{l}\text { Multivariate HR } \\
(95 \% \mathrm{CI})^{\mathrm{c}}\end{array}$ \\
\hline Current smoking (yes vs no) & $1.48(1.18-1.86)$ & $1.67(1.29-2.16)$ & $2.79(1.63-4.77)$ & $3.00(1.70-5.28)$ \\
\hline $\operatorname{BMI}\left(\mathrm{kg} / \mathrm{m}^{2}\right)$ & $1.02(0.99-1.05)$ & $0.99(0.95-1.03)$ & $0.99(0.94-1.05)$ & $0.97(0.92-1.03)$ \\
\hline Systolic BP (10 mm Hg) & $1.09(1.04-1.15)$ & $1.06(1.01-1.12)$ & $1.13(1.02-1.26)$ & $1.15(1.03-1.29)$ \\
\hline Total cholesterol (mmol/l) & $1.17(1.07-1.28)$ & $1.16(1.04-1.31)$ & $0.95(0.78-1.16)$ & $0.84(0.66-1.06)$ \\
\hline HDL cholesterol (mmol/l) & $0.45(0.31-0.67)$ & $0.45(0.29-0.72)$ & $0.48(0.24-0.97)$ & $0.84(0.36-1.96)$ \\
\hline Triglycerides (1 log-unit) & $2.79(1.66-4.68)$ & $1.16(0.55-2.46)$ & $4.56(1.32-15.8)$ & $5.59(0.94-33.1)$ \\
\hline
\end{tabular}

a Only the studies in which the number of cardiovascular deaths was at least four; ${ }^{b}$ Adjusted for age and cohort; ${ }^{c}$ Multivariate models include, in addition to age and cohort, all the risk factors listed in the table; in analyses of men the Helsinki Policemen study was excluded somewhat less marked (fasting insulin, 1.6-2.4 in men and 1.6-2.3 in women; 2 -h insulin, $2.3-3.4$ in men and $2.0-2.5$ in women).

The age- and cohort-adjusted Spearman's correlation coefficients for the association of fasting and 2-h plasma insulin and HOMA-IR with other continuous risk factors in men and women of the pooled study population are given in Table 3. Triglycerides, BMI and glucose variables had strong positive correlations; systolic blood pressure had somewhat weaker positive correlations; and total cholesterol had only weak positive correlations with fasting and 2-h insulin and HOMA-IR. Furthermore, HDL cholesterol had strong inverse correlations with both fasting and 2-h insulin, as well as with HOMA-IR. The correlations between fasting insulin and HOMA-IR were almost perfect. The correlations between 2-h insulin and 2-h glucose were clearly stronger than the correlations between fasting insulin and fasting glucose. There was no association between smoking status and fasting or 2-h insulin; the age-, cohort- and BMI-adjusted prevalences of current smoking, comparing subjects in the highest quartile with those in the combined lower quartiles, were $32.5 \%$ vs $33.5 \%$ (NS) in men and $22.5 \%$ vs $21.8 \%$ (NS) in women in these fasting insulin categories, and $30.7 \%$ vs $28.9 \%$ (NS) in men and $22.4 \%$ vs $21.7 \%$ (NS) in women in the corresponding 2 -h insulin categories.

During the follow-up, which had a median duration of 8.8 years, 362 men and 70 women died from CVD (Table 1). Restricting the data analyses in women to the studies where there were at least four CVD deaths in women reduced the total number of CVD deaths in women to 68 . Because 2-h insulin data were available only from a subsample of 472 subjects (230 men and 242 women) in the Hoorn Study, analyses dealing with 2-h insulin were based on 295 cardiovascular deaths in men and 33 cardiovascular deaths in women.

The associations of conventional risk factors with cardiovascular mortality were assessed by calculating univariate (age- and cohort-adjusted) and multivariate Cox model HRs and 95\% CIs (Table 4). In univariate 
a

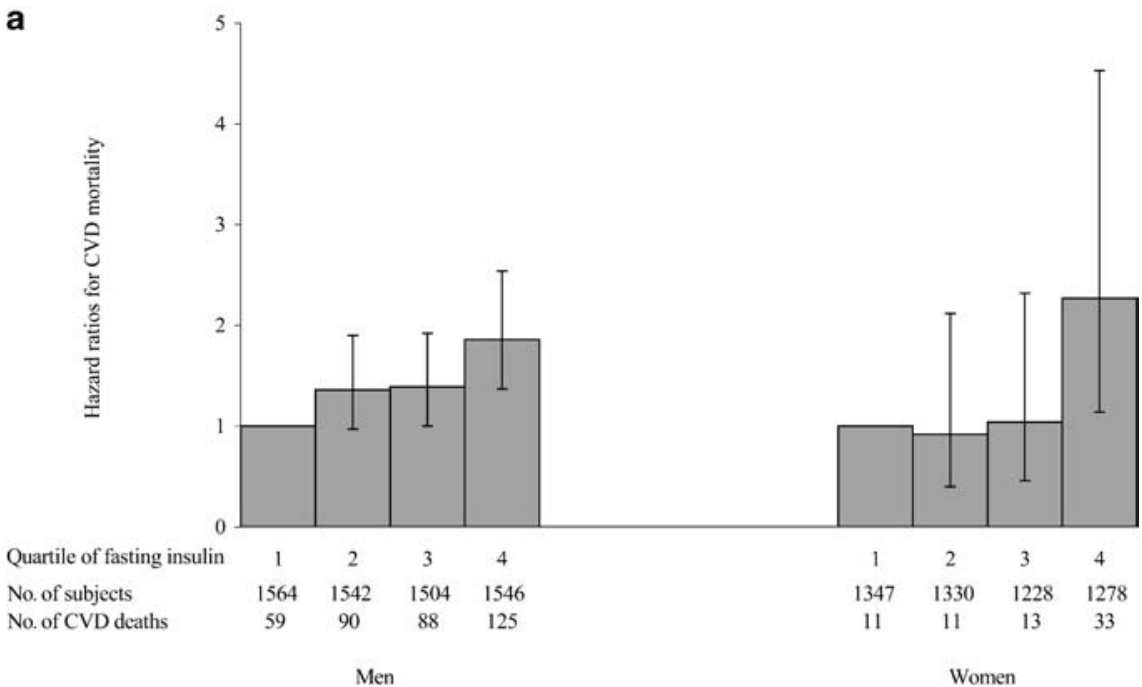

b

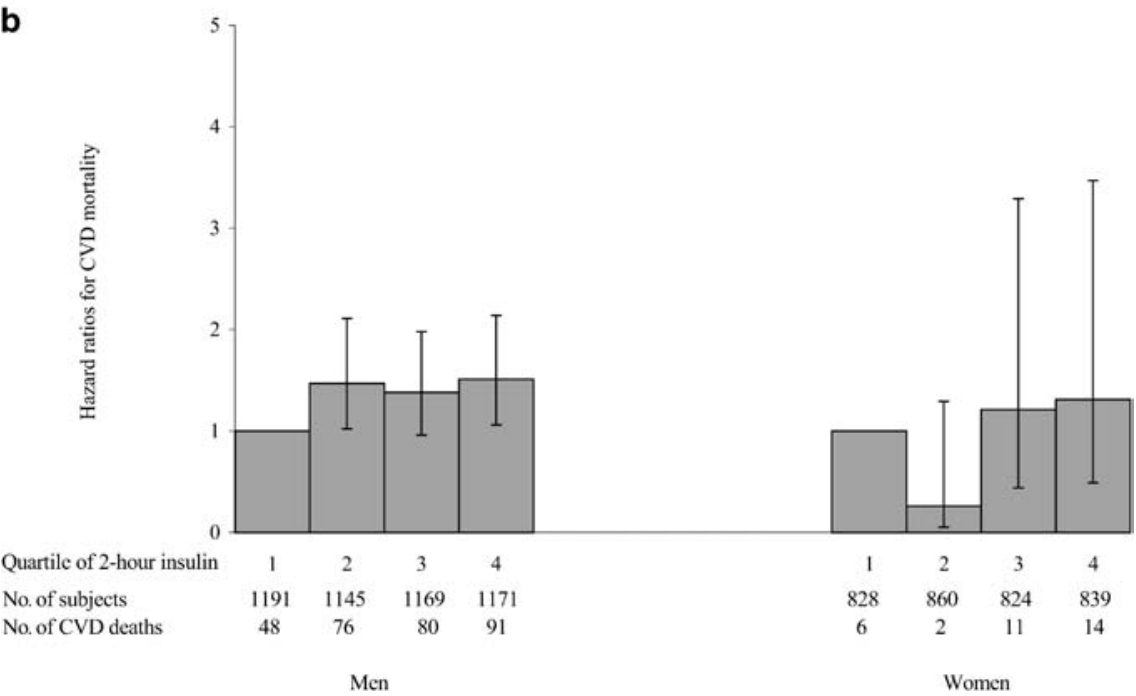

Fig. 1. Age- and cohort-adjusted hazard ratios (95\% CI) for cardiovascular mortality according to sex in pooled DECODE study cohorts by quartiles of fasting insulin (a) and 2-h insulin (b), with the lowest quartile as a reference. The $p$ values for the trends were 0.001 in men and 0.008 in women for fasting insulin, and 0.117 in men and 0.197 in women for 2-h insulin

analyses, positive associations with cardiovascular mortality were found for smoking, systolic blood pressure, 2-h glucose, total cholesterol and triglycerides in men, and for smoking, systolic blood pressure and triglycerides in women. Inverse associations were observed in both men and women between HDL cholesterol and cardiovascular mortality. With multivariate adjustment the association between triglycerides and cardiovascular mortality became non-significant in both men and women, and the same applied to the association between HDL cholesterol and cardiovascular mortality in women.

Figure 1 shows Cox model HRs and 95\% CIs for cardiovascular mortality in men and women, adjusted for age and cohort, in the pooled data by quartiles of fasting insulin and 2-h insulin, with the lowest quartile as a reference. In both sexes there was a significant increase in cardiovascular mortality with increasing fasting insulin levels. Curvilinearity of these associations was tested but could not be confirmed ( $p$ values $>0.10$ in log-likelihood ratio tests). No significant increase in cardiovascular mortality with increasing 2-h insulin levels was observed in either men or women.

Meta-analyses of the association of fasting insulin, HOMA-IR and 2-h insulin with cardiovascular mortality, comparing the highest quartile with combined lower quartiles, are summarised in Table 5. The HRs and $95 \%$ CIs are shown with three levels of adjustment for other variables: Model 1, with adjustment for age and smoking; Model 2, with additional adjustment for BMI, systolic blood pressure, fasting and 2-h glucose, and total cholesterol; and Model 3, with additional adjustment for HDL cholesterol and triglycerides (in ten studies of men and seven studies of women). The statistics for study-to-study variation in effect size show that there was no significant heterogeneity in the outcomes among populations included in the meta-analyses (all $p$ values $>0.10$ ). 
Table 5. Meta-analyses of the association of fasting insulin, HOMA-IR and 2-h insulin (the highest quartile vs quartiles 1-3) with cardiovascular mortality, expressed as overall hazard ratios (HRs) and their 95\% CIs, shown according to sex

Overall HR $(95 \% \mathrm{CI})$

Fasting insulin

Men (eleven studies)

Model $1^{\mathrm{b}}$

Model $2^{c}$

Model 3 (ten studies) ${ }^{\mathrm{d}}$
$1.58(1.26-1.97)$

$1.53(1.19-1.97)$

$1.54(1.16-2.03)$

Non-specific insulin assay (seven studies)

Model 1

Model 2

$1.46(1.12-1.92)$

$1.37(1.01-1.87)$

Specific insulin assay (four studies)

Model 1

Model 2

$1.82(1.25-2.66)$

$1.89(1.24-2.88)$

Women (seven studies)

Model 1

Model 2

Model 3
$2.64(1.54-4.51)$

$2.72(1.51-4.89)$

2.66 (1.45-4.90)
HOMA-IR

$1.59(1.27-1.98)$

$1.53(1.20-1.97)$

$1.58(1.20-2.09)$

$1.43(1.09-1.87)$

$1.34(0.98-1.81)$

$1.94(1.33-2.82)$

$1.99(1.31-3.04)$

$2.40(1.37-4.20)$

$2.41(1.32-4.39)$

$2.35(1.27-4.37)$ 2-h insulin ${ }^{\mathrm{a}}$

$1.28(0.99-1.66)$

$1.07(0.79-1.45)$

$0.85(0.60-1.21)$

$1.19(0.89-1.61)$

$0.99(0.70-1.40)$

$1.59(0.95-2.68)$

$1.42(0.75-2.69)$

$1.87(0.87-4.02)$

$1.28(0.52-3.15)$

$1.36(0.53-3.45)$ a 2-h insulin data were not available from the Cremona Study; in the Hoorn Study, 2-h insulin data were available only from a subsample of 472 subjects ( 230 men, 242 women); b Adjustment for age and smoking; ${ }^{c}$ Model 1 plus additional adjustment for BMI, systolic BP, fasting glucose (except in HOMA-
IR analyses), 2-h glucose and total cholesterol; d Model 2 plus additional adjustment for HDL cholesterol and triglycerides; Helsinki Policemen Study excluded; e Only the studies in which the number of cardiovascular deaths was at least four. HOMAIR, homeostasis model assessment of insulin resistance
With Model 1 adjustment, the overall HR for cardiovascular mortality in the highest quartile vs combined lower quartiles for fasting insulin was significantly increased in both men and women and was not further attenuated with Model 2 and Model 3 adjustments. The results for HOMA-IR were almost similar, as expected. The overall HR for cardiovascular mortality in the highest quartile for 2-h insulin, however, was not statistically significant and became markedly reduced with Model 2 and Model 3 adjustments.

We assessed the possible influence of the type of insulin assay (non-specific vs specific) by calculating overall HRs for cardiovascular mortality in the highest quartile vs combined lower quartiles for fasting insulin, HOMA-IR, and 2-h insulin with Model 1 and Model 2 adjustments in the seven studies in men which used non-specific assays, and in the four studies in men which used specific assays (Table 5). The results were not substantially influenced by the type of assay used.

To assess the association of fasting and 2-h insulin with cardiovascular mortality in a comparable way over a wider range of the distributions of the two variables (without log transformation) we calculated HRs for individual studies and carried out meta-analyses for sex- and cohort-specific interquartile ranges for fasting and 2-h insulin with Model 2 adjustment (Fig. 2). There was a wide variation in HRs between studies. In men, the overall HRs for fasting insulin and 2-h insulin were almost similar and were statistically significant. In women, the overall HR for fasting insulin was statistically significant, whereas the overall HR for 2-h insulin was not.

\section{Discussion}

Our meta-analyses, based on data from eleven European prospective studies, showed a statistically significant positive association between fasting insulin and cardiovascular mortality. It was similar in both nondiabetic men and women, and was independent of plasma glucose and other cardiovascular risk factors. There was an increased CVD risk in subjects in the highest quartile of fasting insulin as compared with those in combined lower quartiles, and also a linear increase in CVD risk over the fasting insulin distribution. The association between high 2 -h insulin and CVD risk was weaker and was not statistically significant in the highest vs combined lower quartiles comparison. However, meta-analyses carried out using a comparable measure for the increment in fasting and 2-h insulin, sex- and cohort-specific interquartile

Fig. 2. Individual and overall hazard ratios (95\% CI) for cardiovascular mortality, calculated for sex- and cohort-specific interquartile ranges, for fasting insulin in men (a) and women (b) and for 2-h insulin in men (c) and women (d) of the DECODE study cohorts, adjusting for age, smoking, BMI, systolic blood pressure, fasting glucose, 2-h glucose and total cholesterol 


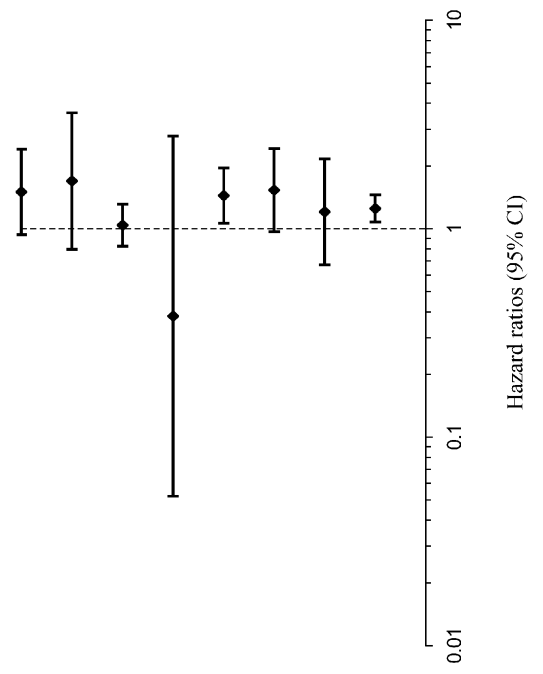

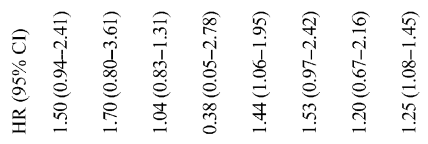

○.

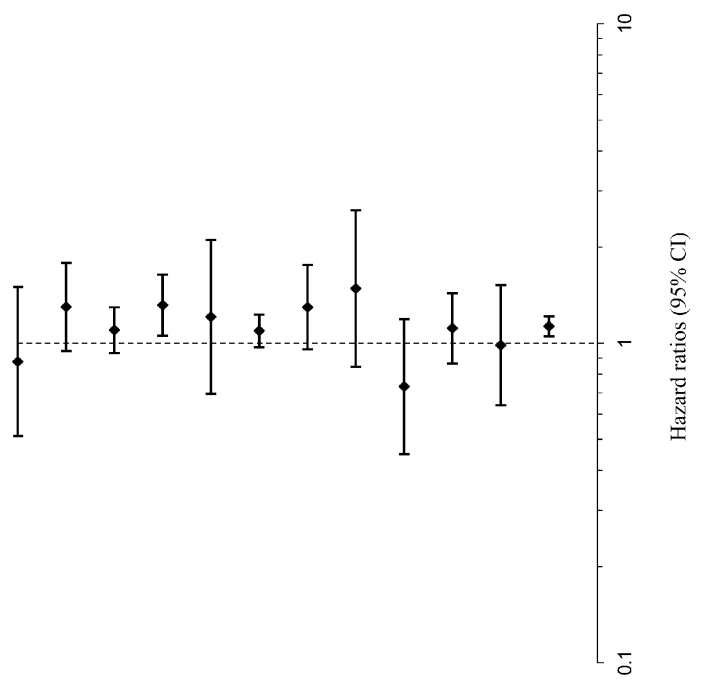

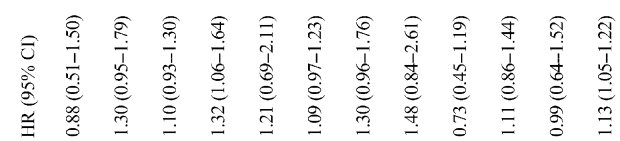

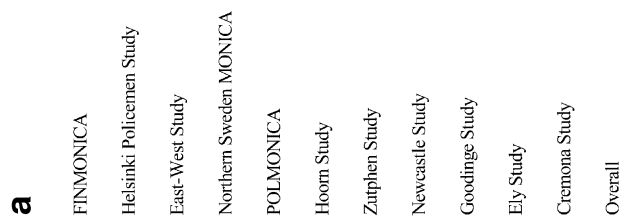

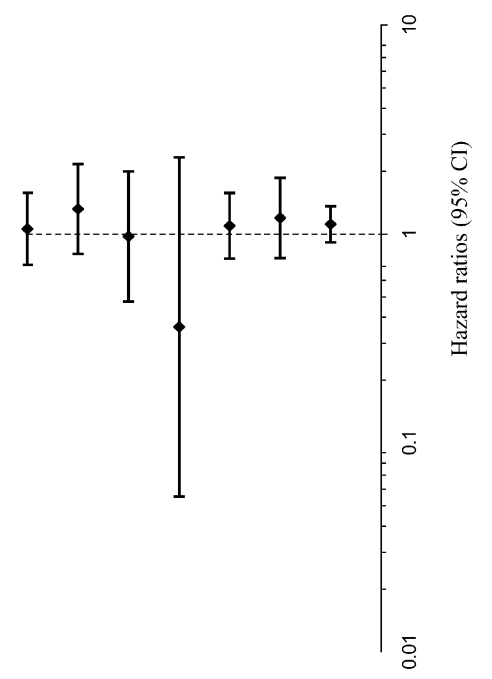

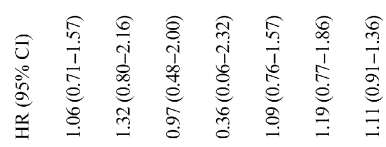

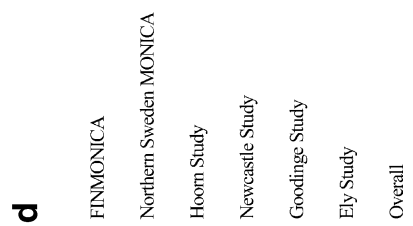

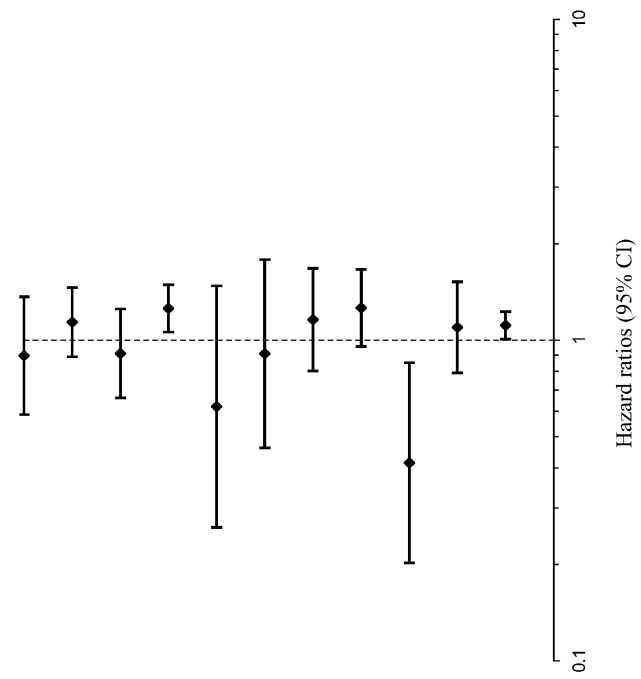

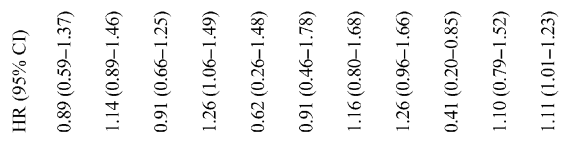

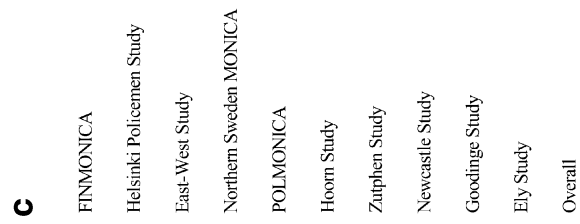


range (approximately $50 \mathrm{pmol} / \mathrm{l}$ for fasting insulin and $200 \mathrm{pmol} / \mathrm{l}$ for 2 -h insulin), demonstrated a more similar weak positive association of fasting and 2-h insulin with CVD risk. The overall HR for the interquartile range increment in fasting insulin was 1.13 in men and 1.25 in women, and for the respective increment in 2-h insulin, 1.11 in both men and women. These overall hazard ratios are of the same magnitude as the summary relative risks reported from a previous metaanalysis of the association between plasma insulin and CVD risk based on studies identified from the literature [34]; in seven studies in Caucasoid populations the summary relative risk of CVD per increment of $50 \mathrm{pmol} / \mathrm{l}$ in fasting insulin was 1.18 , and per increment of $250 \mathrm{pmol} / \mathrm{l}$ in non-fasting insulin it was 1.16 , increments corresponding to the respective interquartile ranges in the Dutch population.

HOMA-IR score, developed from the mathematical modelling of fasting plasma glucose and insulin concentrations [31], has recently been used as a surrogate measure of insulin resistance in some epidemiological studies examining the relationship between insulin resistance and CVD risk [12, 13, 16]. The results of studies that validate HOMA-IR score when compared with direct measures of insulin resistance are, however, conflicting. Some studies have reported a stronger correlation of HOMA-IR than of fasting insulin with directly measured insulin resistance $[31,35]$, whereas in other studies HOMA-IR was not found to be better than fasting insulin in this respect $[36,37]$. In our study cohorts, the Spearman's rank correlation coefficients between HOMA-IR and fasting insulin were $\geq 0.98$. Therefore, it is not surprising that in our meta-analyses, the associations of HOMA-IR and fasting insulin with cardiovascular mortality were almost identical.

In some recent studies, plasma concentrations of proinsulin-like molecules have been shown to be associated with an increased risk of CVD, even more strongly than plasma concentration of intact insulin, and also independently of intact insulin [14, 21, 38]. In non-diabetic subjects, proinsulin and its split products account for about $10 \%$ of all immunoreactive insulinlike molecules [39]. Thus, the use of insulin assays that cross-react with proinsulin-like molecules might influence the association between insulin and CVD risk. In our meta-analyses the overall HRs for CVD death in the highest vs combined lower quartiles of fasting insulin, HOMA-IR and 2-h insulin were, however, rather similar in studies using assays specific for intact insulin and in those using non-specific methods.

It is well known that due to intra-individual shortterm and long-term variation in the levels of biological risk factors, their measurement on a single occasion, as was done in all of our studies, does not classify individuals accurately with regard to their 'usual' risk factor levels. Because of the fluctuations in the measured values of risk factors, prospective studies based on single risk factor measurements tend to underestimate the real association between the 'usual' level of a risk factor and the disease rate during the follow-up, a phenomenon called the "regression dilution" effect [40, 41]. The intra-individual variation in plasma insulin levels has been shown to be relatively large [42]; in Dutch subjects with normal glucose tolerance the ratio of the standard deviation of the test-retest difference to the median of plasma insulin measured with a specific method was found to be 23/76 pmol/1 for fasting insulin and 190/303 pmol/1 for 2-h insulin. These test-retest differences were predominantly determined by a real biological variation, with analytical variation making only a minor contribution. In our study the regression dilution effect may have led to a substantial underestimation of the true association between insulin and CVD risk, even more so with 2-h insulin than with fasting insulin because of the much larger biological variation in 2-h insulin. This may explain why 2 -h insulin showed a weaker association with CVD risk than fasting insulin in our meta-analyses. However, to some extent the weaker association of 2-h insulin with CVD risk could also be explained by a smaller power of analyses on 2-h insulin; in one study 2-h insulin data were only available from a subsample and in another study data were not available at all, which led to a substantial reduction in the number of endpoints.

In univariate and multivariate analyses carried out in the pooled DECODE study populations the associations between conventional risk factors and CVD mortality corresponded largely with expectations (Table 4). In the meta-analyses of the associations of fasting and 2-h insulin with CVD mortality, multivariate adjustments, including the conventional risk factors and also the core components of the metabolic syndrome (BMI, glucose, blood pressure, triglycerides and HDL cholesterol), resulted only in a modest attenuation of the association between fasting insulin and CVD mortality. The association between 2-h insulin and CVD mortality was, however, substantially reduced by the adjustment for other risk factors, particularly in the analysis of the highest vs combined lower quartiles. It is possible that this greater effect of multivariate adjustment on the association between 2-h insulin and CVD mortality is mainly explained by the strong correlation between 2-h insulin and 2-h glucose levels. In the interpretation of the outcomes of these meta-analyses it is important to note that in addition to the core components of the metabolic syndrome included in our multivariate adjustments, there are also other factors, such as thrombogenic factors [43] and inflammation markers [44], that are strongly linked with hyperinsulinaemia and insulin resistance and, on the other hand, with increased CVD risk. The possibility that an unaccounted residual confounding by such factors had an effect on our results cannot be excluded.

The finding in our study and in a number of previously published individual prospective studies $[1,2,3$, 
$5,6,7,9,10,12,13,15,16]$ that the association between plasma insulin level and CVD risk is independent of other risk factors is even compatible with the view that insulin, through some mechanism, could have a direct effect on the development of atherosclerosis. In vitro studies of the cells of the arterial wall have suggested that insulin could stimulate smooth cell proliferation and binding of LDL cholesterol to smooth muscle cells, fibroblasts and monocytes [45]. Animal experiments trying to produce atherosclerosislike arterial lesions by the administration of exogenous insulin have, however, produced conflicting and unconvincing results [45, 46, 47]. Furthermore, longterm clinical trials in subjects with Type 2 diabetes, testing the effects of different treatment modalities on the occurrence of cardiovascular complications, have not demonstrated any deleterious effect of insulin treatment $[48,49]$. An alternative explanation for the independent association between insulin and CVD risk could be that the underlying insulin resistance itself or some unidentified background factor leading to insulin resistance and hyperinsulinaemia directly enhances the development of atherosclerotic vascular disease. There is evidence from cross-sectional studies that insulin resistance measured by direct methods is associated with ultrasonographically measured intimamedial thickening of the arterial walls, even independently of the risk factors belonging to the metabolic syndrome $[50,51,52]$. Two prospective studies have also reported findings suggesting that directly measured insulin resistance predicts CVD risk [53, 54].

Our study has several limitations. One limitation is that the study populations included in our meta-analyses were not identified in an orthodox way, by a search of published studies in the literature. Instead they were identified among studies participating in the collaborative DECODE Study which was originally started for other purposes. We have not, however, been able to identify in this set of studies any elements of selection bias or heterogeneity that might have distorted the association between insulin and CVD risk. Another limitation is that baseline risk factor measurements were not carried out by methods standardised between the study centres. With regard to plasma insulin determinations, we tried to overcome the diversity in the methods by the use of sex- and cohort-specific cut-offs for the fasting and 2-h insulin in analyses based on insulin quartiles and interquartile ranges. In any case, differences in the methods used in risk factor measurements may have led to some dilution in the risk estimates. A further limitation of our study is that we could not exclude individuals with prevalent CVD, because baseline data collection was not uniform in that respect. The associations between risk factors and CVD events tend to become attenuated to some extent after the clinical manifestation of CVD for several reasons (survival bias, treatment effects and the strong relationship of established CVD to the risk of recurrent events). Thus, inclusion of people with prevalent CVD may have led to some attenuation of the association between insulin and CVD risk. An important strength of our meta-analyses is that instead of using aggregate data we were able to use data on individuals, thus reducing the risk of spurious results [55].

Our finding that the fasting plasma insulin concentration, more consistently than the 2-h insulin concentration, shows a positive correlation with CVD risk independently of other risk factors is of relevance in relation to the proposal by the European Group for the Study of Insulin Resistance (EGIR) [25] that fasting hyperinsulinaemia could be used in the definition of the metabolic syndrome as a proxy for insulin resistance measured by the euglycaemic insulin clamp, as originally proposed by the WHO Consultation [32]. According to the EGIR, insulin-resistant people could be defined as those with fasting plasma insulin levels above the highest plasma insulin quartile cut-off in the non-diabetic background population, the cut-off used in our highest vs combined lower quartiles meta-analyses. Fasting plasma insulin concentration is, however, not a perfect surrogate marker for insulin resistance. The correlation of fasting plasma insulin with directly measured insulin resistance is about 0.60 and thus fasting plasma insulin explains only about $40 \%$ of the variance in insulin resistance $[36,56]$. The correlation between 2 -h insulin and directly measured insulin resistance is of a similar magnitude $[36,56]$. These correlations are based on single plasma insulin measurements and, as discussed earlier, such measurements do not always give a correct estimate of the 'usual' plasma insulin level and, consequently, insulin resistance for an individual. Furthermore, correlations of plasma insulin levels and directly measured indices of insulin resistance with other risk factor components of the metabolic syndrome are not limited to the coexistence of high levels of these risk factors with high insulin levels and insulin resistance but extend over a wider range of their distributions [57, 58]. The findings of the present study indicate that the excess CVD risk is not confined to the highest quartiles of plasma insulin levels with CVD risk but rather is more linear in character. Therefore, the inclusion of hyperinsulinaemia in the definition of the metabolic syndrome may not make a major contribution in the prediction of CVD risk. We have, in fact, shown in another study based on the same eleven DECODE studies that inclusion of hyperinsulinaemia (highest fasting insulin quartile) in the definition of the metabolic syndrome, in addition to the presence of two or more, or three or more of its other core components, resulted only in a modest improvement in the prediction of the CVD risk, with a slightly improved specificity but a reduced sensitivity and positive predictive value [59].

Poor inter-laboratory reproducibility of plasma insulin measurements remains the greatest problem in 
the use of insulin in the prediction of CVD risk, as was pointed out by the American Diabetes Association's Task Force on standardisation of the insulin assay [60]. With the introduction of assays specific for intact insulin, the within-laboratory analytical precision has improved, but even with these assays, the use of the same assay kits in different laboratories does not always ensure comparable measurements. As emphasised by the Task Force, a central reference laboratory, ongoing sample exchange and rigorous quality control would be needed to improve and maintain the reliability of plasma insulin measurements.

In conclusion, our meta-analyses of prospective data from eleven DECODE study populations have demonstrated that hyperinsulinaemia, defined by the highest quartile cut-off for fasting insulin, was significantly and similarly associated with cardiovascular mortality in non-diabetic men and women, independently of other risk factors. In the corresponding highest vs combined lower quartiles meta-analyses, the associations between high 2-h insulin and cardiovascular mortality were weaker and non-significant. Weak positive associations of fasting and 2-h insulin with cardiovascular mortality over interquartile ranges were, however, more similar.

Acknowledgements. This analysis was carried out with the help of grants from Novartis Pharma (Basel, Switzerland), from AstraZeneca R\&D (Mölndal, Sweden) and from the Finnish Academy (grants 46558, 76502, 77618, 204274 and 205657). The DECODE Study was initially funded by Novo Nordisk (Bagsvaerd, Denmark).

\section{Organisation}

The DECODE Study (Diabetes Epidemiology: Collaborative analysis Of Diagnostic Criteria in Europe) was undertaken in 1997 on the initiative of the European Diabetes Epidemiology Group.

Studies and investigators in this collaborative study were:

\section{Finland}

The East-West Finland Study: A. Nissinen, J. Pekkanen, J. Tuomilehto, Department of Epidemiology and Health Promotion, National Public Health Institute, Helsinki

FINMONICA: J. Tuomilehto, P. Jousilahti, J. Lindström, Department of Epidemiology and Health Promotion, National Public Health Institute, Helsinki

The Helsinki Policemen Study: M. Pyörälä, K. Pyörälä, Department of Medicine, University of Kuopio, Kuopio

\section{Italy}

The Cremona Study: G. Gallus, Institute of Medical Statistics, University of Milan, and M. P. Garancini, Quality Direction, S. Raffaele Institute, Milan

\section{The Netherlands}

The Hoorn Study: L. M. Bouter, J. M. Dekker, R. J. Heine, G. Nijpels, C. D. A. Stehouwer, Institute for Research in Extramural Medicine, Vrije Universiteit Medical Center, Amsterdam
The Zutphen Elderly Study: E. J. M. Feskens, D. Kromhout, National Institute of Public Health and the Environment, Bitlhoven

\section{Poland}

POLMONICA (Krakow): A. Pajak, Department of Clinical Epidemiology and Population Studies, Institute of Public Health, Collegium Medicum, Jagellonian University, Krakow

\section{Sweden}

Northern Sweden MONICA (three cohorts): M. Eliasson, B. Stegmayr, V. Lundberg, Department of Medicine, University of Umeå, Umeå

\section{United Kingdom}

Isle of Ely Diabetes Project: N. J. Wareham, Department of Public Health and Primary Care, University of Cambridge, Cambridge

Newcastle Heart Project: N. Unwin, N. Ahmad, K. G. M. M. Alberti, L. Hayes, Department of Medicine and Epidemiology and Public Health, University of Newcastle, Newcastle

The Goodinge Study: J. S. Yudkin, M. Gould, A. Haines, R. W. Morris, Centre for Diabetes and Cardiovascular Risk, and Department of Primary Health Care, University College London Medical School, London

\section{Secretariat}

K. Borch-Johnsen, Steno Diabetes Center, Gentofte, Denmark

J. Eriksson, Q. Qiao, J. Tuomilehto, Department of Epidemiology and Health Promotion, National Public Health Institute, Helsinki, Finland

\section{Data analysis}

G. Hu, Q. Qiao, Diabetes and Genetic Epidemiology Unit, Department of Epidemiology and Health Promotion, National Public Health Institute, Helsinki, Finland

K. Pyörälä, Department of Medicine, University of Kuopio, Finland

\section{Writing committee}

G. Hu, Q. Qiao, J. Tuomilehto, Diabetes and Genetic Epidemiology Unit, Department of Epidemiology and Health Promotion, National Public Health Institute, Helsinki, and Department of Public Health, University of Helsinki, Finland

M. Eliasson, Department of Medicine, Sunderby Hospital, Luleå, and Umeå University, Sweden

E. J. M. Feskens, Department of Nutrition and Health, National Institute of Public Health and the Environment, Bitlhoven, the Netherlands

K. Pyörälä, Department of Medicine, University of Kuopio, Finland

\section{References}

1. Pyörälä K (1979) Relationship of glucose tolerance and plasma insulin to the incidence of coronary heart disease: results from two population studies in Finland. Diabetes Care 2:131-141

2. Welborn TA, Wearne K (1979) Coronary heart disease incidence and cardiovascular mortality in Busselton with reference to glucose and insulin concentrations. Diabetes Care 2:154-160 
3. Ducimetiere P, Eschwege E, Papoz L, Richard JL, Claude JR, Rosselin G (1980) Relationship of plasma insulin levels to the incidence of myocardial infarction and coronary heart disease mortality in a middle-aged population. Diabetologia 19:205-210

4. Yarnell JW, Sweetnam PM, Marks V, Teale JD, Bolton CH (1994) Insulin in ischaemic heart disease: are associations explained by triglyceride concentrations? The Caerphilly Prospective Study. Br Heart J 71:293-296

5. Møller LF, Jespersen J (1995) Fasting serum insulin levels and coronary heart disease in a Danish cohort: 17-year follow-up. J Cardiovasc Risk 2:235-240

6. Després JP, Lamarche B, Mauriege P et al. (1996) Hyperinsulinemia as an independent risk factor for ischemic heart disease. N Engl J Med 334:952-957

7. Perry IJ, Wannamethee SG, Whincup PH, Shaper AG, Walker MK, Alberti KG (1996) Serum insulin and incident coronary heart disease in middle-aged British men. Am J Epidemiol 144:224-234

8. Folsom AR, Szklo M, Stevens J, Liao F, Smith R, Eckfeldt JH (1997) A prospective study of coronary heart disease in relation to fasting insulin, glucose, and diabetes. The Atherosclerosis Risk in Communities (ARIC) Study. Diabetes Care 20:935-942

9. Pyörälä M, Miettinen H, Laakso M, Pyörälä K (1998) Hyperinsulinemia predicts coronary heart disease risk in healthy middle-aged men: The 22-Year Follow-up Results of the Helsinki Policemen Study. Circulation 98:398-404

10. Lempiäinen $\mathrm{P}$, Mykkänen L, Pyörälä K, Laakso M, Kuusisto J (1999) Insulin resistance syndrome predicts coronary heart disease events in elderly nondiabetic men. Circulation 100:123-128

11. Lakka HM, Lakka TA, Tuomilehto J, Sivenius J, Salonen JT (2000) Hyperinsulinemia and the risk of cardiovascular death and acute coronary and cerebrovascular events in men: the Kuopio Ischaemic Heart Disease Risk Factor Study. Arch Intern Med 160:1160-1168

12. Hanley AJ, Williams K, Stern MP, Haffner SM (2002) Homeostasis model assessment of insulin resistance in relation to the incidence of cardiovascular disease: the San Antonio Heart Study. Diabetes Care 25:1177-1184

13. Hedblad B, Nilsson P, Engström G, Berglund G, Janzon L (2002) Insulin resistance in non-diabetic subjects is associated with increased incidence of myocardial infarction and death. Diabet Med 19:470-475

14. Zethelius B, Byberg L, Hales CN, Lithell H, Berne C (2002) Proinsulin is an independent predictor of coronary heart disease: report from a 27 -year follow-up study. Circulation 105:2153-2158

15. Nilsson P, Nilsson JÄ, Hedblad B, Eriksson KF, Berglund G (2003) Hyperinsulinaemia as long-term predictor of death and ischaemic heart disease in nondiabetic men: The Malmö Preventive Project. J Intern Med 253:136-145

16. Welin L, Bresäter LE, Eriksson H, Hansson PO, Welin C, Rosengren A (2003) Insulin resistance and other risk factors for coronary heart disease in elderly men. The Study of Men Born in 1913 and 1923. Eur J Cardiovasc Prev Rehabil 10:283-288

17. Collins VR, Dowse GK, Zimmet PZ et al. (1993) Serum insulin and ECG abnormalities suggesting coronary heart disease in the populations of Mauritius and Nauru: cross-sectional and longitudinal associations. J Clin Epidemiol 46:1373-1393

18. Orchard TJ, Eichner J, Kuller LH, Becker DJ, McCallum LM, Grandits GA (1994) Insulin as a predictor of coronary heart disease: interaction with apolipoprotein E phenotype. A report from the Multiple Risk Factor Intervention Trial. Ann Epidemiol 4:40-45
19. Ferrara A, Barrett-Connor EL, Edelstein SL (1994) Hyperinsulinemia does not increase the risk of fatal cardiovascular disease in elderly men or women without diabetes: the Rancho Bernardo Study, 1984-1991. Am J Epidemiol 140:857-869

20. Lindberg O, Tilvis RS, Strandberg TE et al. (1997) Elevated fasting plasma insulin in a general aged population: an innocent companion of cardiovascular diseases. J Am Geriatr Soc 45:407-412

21. Lindahl B, Dinesen B, Eliasson M et al. (1999) High proinsulin concentration precedes acute myocardial infarction in a nondiabetic population. Metabolism 48:1197-1202

22. DeFronzo RA, Jacot E, Jequier E, Maeder E, Wahren J, Felber JP (1981) The effect of insulin on the disposal of intravenous glucose. Results from indirect calorimetry and hepatic and femoral venous catheterization. Diabetes 30:1000-1007

23. Reaven GM (1988) Banting lecture 1988. Role of insulin resistance in human disease. Diabetes 37:1595-1607

24. Liese AD, Mayer-Davis EJ, Haffner SM (1998) Development of the multiple metabolic syndrome: an epidemiologic perspective. Epidemiol Rev 20:157-172

25. Balkau B, Charles MA (1999) Comment on the provisional report from the WHO consultation. European Group for the Study of Insulin Resistance (EGIR). Diabet Med 16:442-443

26. DECODE Study Group (1998) Will new diagnostic criteria for diabetes mellitus change phenotype of patients with diabetes? Reanalysis of European epidemiological data. DECODE Study Group on behalf of the European Diabetes Epidemiology Study Group. BMJ 317:371-375

27. DECODE Study Group (1999) Is fasting glucose sufficient to define diabetes? Epidemiological data from 20 European studies. The DECODE-study group. European Diabetes Epidemiology Group. Diabetes Epidemiology: Collaborative analysis of Diagnostic Criteria in Europe. Diabetologia 42:647-654

28. DECODE Study Group (1999) Glucose tolerance and mortality: comparison of WHO and American Diabetes Association diagnostic criteria. The DECODE study group. European Diabetes Epidemiology Group. Diabetes Epidemiology: Collaborative analysis Of Diagnostic criteria in Europe. Lancet 354:617-621

29. DECODE Study Group (2001) Glucose tolerance and cardiovascular mortality: comparison of fasting and 2-hour diagnostic criteria. Arch Intern Med 161:397-405

30. DECODE Study Group (2003) Gender difference in all-cause and cardiovascular mortality related to hyperglycaemia and newly-diagnosed diabetes. Diabetologia 46:608-617

31. Matthews DR, Hosker JP, Rudenski AS, Naylor BA, Treacher DF, Turner RC (1985) Homeostasis model assessment: insulin resistance and beta-cell function from fasting plasma glucose and insulin concentrations in man. Diabetologia 28:412-419

32. World Health Organization (1999) Definition, diagnosis and classification of diabetes mellitus and its complications. Part 1: diagnosis and classification of diabetes mellitus. Report of a WHO Consultation, WHO, Geneva

33. Fleiss JL (1993) The statistical basis of meta-analysis. Stat Methods Med Res 2:121-145

34. Ruige JB, Assendelft WJ, Dekker JM, Kostense PJ, Heine RJ, Bouter LM (1998) Insulin and risk of cardiovascular disease: a meta-analysis. Circulation 97:996-1001

35. Bonora E, Targher G, Alberiche M et al. (2000) Homeostasis model assessment closely mirrors the glucose clamp technique in the assessment of insulin sensitivity: studies in subjects with various degrees of glucose tolerance and insulin sensitivity. Diabetes Care 23:57-63 
36. Yeni-Komshian H, Carantoni M, Abbasi F, Reaven GM (2000) Relationship between several surrogate estimates of insulin resistance and quantification of insulin-mediated glucose disposal in 490 healthy nondiabetic volunteers. Diabetes Care 23:171-175

37. McAuley KA, Williams SM, Mann JI et al. (2001) Diagnosing insulin resistance in the general population. Diabetes Care 24:460-464

38. Yudkin JS, May M, Elwood P, Yarnell JW, Greenwood R, Davey Smith G (2002) Concentrations of proinsulin like molecules predict coronary heart disease risk independently of insulin: prospective data from the Caerphilly Study. Diabetologia 45:327-336

39. Nagi DK, Knowler WC, Mohamed-Ali V, Bennett PH, Yudkin JS (1998) Intact proinsulin, des 31,32 proinsulin, and specific insulin concentrations among nondiabetic and diabetic subjects in populations at varying risk of type 2 diabetes. Diabetes Care 21:127-133

40. MacMahon S, Peto R, Cutler J et al. (1990) Blood pressure, stroke, and coronary heart disease. Part 1, Prolonged differences in blood pressure: prospective observational studies corrected for the regression dilution bias. Lancet 335:765774

41. Clarke R, Shipley M, Lewington S et al. (1999) mUnderestimation of risk associations due to regression dilution in long-term follow-up of prospective studies. Am J Epidemiol 150:341-353

42. Mooy JM, Grootenhuis PA, de Vries H et al. (1996) Intraindividual variation of glucose, specific insulin and proinsulin concentrations measured by two oral glucose tolerance tests in a general Caucasian population: the Hoorn Study. Diabetologia 39:298-305

43. Juhan-Vague I, Alessi MC (1997) PAI-1, obesity, insulin resistance and risk of cardiovascular events. Thromb Haemost 78:656-660

44. Festa A, D’Agostino R Jr, Howard G, Mykkänen L, Tracy RP, Haffner SM (2000) Chronic subclinical inflammation as part of the insulin resistance syndrome: the Insulin Resistance Atherosclerosis Study (IRAS). Circulation 102:42-47

45. Stout R (1990) Insulin and atheroma. 20-yr perspective. Diabetes Care 13:631-654

46. Sato Y, Shiraishi S, Oshida Y, Ishiguro T, Sakamoto N (1989) Experimental atherosclerosis-like lesions induced by hyperinsulinism in Wistar rats. Diabetes 38:91-96

47. Nordestgaard BG, Agerholm-Larsen B, Stender S (1997) Effect of exogenous hyperinsulinaemia on atherogenesis in cholesterol-fed rabbits. Diabetologia 40:512-520

48. Knatterud GL, Klimt CR, Levin ME, Jacobson ME, Goldner MG (1978) Effects of hypoglycemic agents on vascular complications in patients with adult-onset diabe- tes. VII. Mortality and selected nonfatal events with insulin treatment. JAMA 240:37-42

49. UK Prospective Diabetes Study Group (1998) Intensive blood-glucose control with sulphonylureas or insulin compared with conventional treatment and risk of complications in patients with type 2 diabetes (UKPDS 33). UK Prospective Diabetes Study (UKPDS) Group. Lancet 352:837-853

50. Laakso M, Sarlund H, Salonen R et al. (1991) Asymptomatic atherosclerosis and insulin resistance. Arterioscler Thromb 11:1068-1076

51. Agewall S, Fagerberg B, Attvall S, Wendelhag I, Urbanavicius V, Wikstrand J (1995) Carotid artery wall intimamedia thickness is associated with insulin-mediated glucose disposal in men at high and low coronary risk. Stroke 26:956-960

52. Howard G, O'Leary DH, Zaccaro D et al. (1996) Insulin sensitivity and atherosclerosis. The Insulin Resistance Atherosclerosis Study (IRAS) Investigators. Circulation 93:1809-1817

53. Yip J, Facchini FS, Reaven GM (1998) Resistance to insulin-mediated glucose disposal as a predictor of cardiovascular disease. J Clin Endocrinol Metab 83:27732776

54. Zethelius B (2002) Proinsulin and insulin sensitivity as predictors of type 2 diabetes mellitus and coronary heart disease-clinical and epidemiological studies with up to 27 years of follow-up. Doctoral thesis, Uppsala University, Sweden

55. Egger M, Davey Smith G, Schneider M (2001) Systematic reviews of observational studies. In: Egger M, Davey Smith G, Altman DG (eds) Systematic reviews in health care. Meta-analysis in context. BMJ Books, London, pp 211-227

56. Laakso M (1993) How good a marker is insulin level for insulin resistance? Am J Epidemiol 137:959-965

57. Mykkänen L, Haffner SM, Rönnemaa T, Bergman RN, Laakso M (1997) Low insulin sensitivity is associated with clustering of cardiovascular disease risk factors. Am J Epidemiol 146:315-321

58. Pyörälä M, Miettinen H, Laakso M, Pyörälä K (2000) Plasma insulin and all-cause, cardiovascular, and noncardiovascular mortality: the 22-year follow-up results of the Helsinki Policemen Study. Diabetes Care 23:1097-1102

59. DECODE Study Group (2004) Prevalence of the metabolic syndrome and its relation to all-cause and cardiovascular mortality in nondiabetic European men and women. Arch Intern Med 164:1066-1076

60. Robbins DC, Andersen L, Bowsher R et al. (1996) Report of the American Diabetes Association's Task Force on standardization of the insulin assay. Diabetes 45:242-256 